\title{
FINAL EXPERTISE AND ATTITUDE TOWARDS VISION SCREENING IN HEALTH CARE PROFES- SIONALS ENROLLED IN THE EUSCREEN PROJECT
}

\author{
Oana Teodosescu, Mihai Mara, Cristina Vladutiu, Simona Cainap, Daniela Rajka \\ UMF Iuliu Hatieganu, Cluj-Napoca
}

\begin{abstract}
AIMS: The aim of the study is to evaluate the knowledge and attitude towards vision screening in health care professionals enrolled in the EUSCREEN project ("Implementation of optimized childhood vision and hearing screening programmes in middle-income countries in Europe"), after the completion of the project.

METHOD: The analysis involved 44 questionnaires, filled out by doctors and nurses who measured visual acuity in children in the EUSCREEN project between January 1st 2018 and December 31st 2019.

RESULTS: The 44 respondents felt confident $(>95 \%)$ in their abilities when it comes to the measurement of visual acuity in children.

Among the main reasons why the study did not include a higher number of preschoolers, the participants to the survey stated: lack of parents' consent (8) or lack of time (2), interest or confidence in the Euscreen project (5); some parents expressed their concern regarding the managing of data confidentiality. Furthermore, some nurses stressed upon the low attendance rate especially in rural kindergartens (4).

The nurses and the doctors felt the supportive materials were helpful in persuading the parents, as well as the teachers' input. Numerous parents did not continue the investigations with an ophthalmological examination, if needed, the main reasons being
\end{abstract}

lack of time, lack of interest in the child's healthcare, lack of financial means or too long waiting lists to ophthalmologists.

In the matter of remuneration of medical staff, $43 \%$ (19) consider it should be made in addition to the salary, meanwhile $34 \%$ (15) consider the screening activity as a compulsory duty of their job.

$70 \%$ (31) respondents claim they will continue the screening activity as they understand it's importance. 50\% (22) feel there should be a periodic (once every 12,24 or 36 months) update of their theoretical notions and practical skills.

DISCUSSION: The respondents consider that a better communication with the parents would have led to a better participation rate and to a better rate of ophthalmological results. Also, when the children are examined in the kindergarten the nurses would prefer the teachers helped by assisting the children. The payment of the medical personnel involved in the screening was preferred by the respondents, as it was considered extra work by the family doctors nurses and by some of the kindergarten nurses. The medical staff feels confident in their measuring technique, only half of them considering that an update would be necessary.

CONCLUSIONS: The EUSCREEN project updated the theoretical notions and practical abilities of the medical staff and made them aware of the importance of early detection of vision problems in children.

\footnotetext{
* Author correspondent: Oana Teodosescu, UMF Iuliu Haţieganu, Cluj-Napoca, e-mail: teodosescuoana@yahoo.com Received: 31.12.2020, accepted: 4.01.2021, published: 10.01.2021

Cite: Teodosescu O, Mara M, Vladutiu C, Cainap S, Rajka D. Final expertise and attitude towards vision screening in health care professionals enrolled in the EUSCREEN project. Journal of School and University Medicine 2020;7(4):17-24
} 
Their answers provide essential directions that a national screening programme should take into account: better communication with the parents; better access to speciality examinations when needed; better coverage in the rural areas, especially in the remote areas; proper reimbursement for the medical staff.

KEYWORDS: visual acuity screening, final survey, amblyopia, feedback

\section{INTRODUCTION}

Amblyopia, a medical condition also referred to as "lazy eye" signifies the reduction of best-corrected visual acuity of one or both eyes that cannot be attributed exclusively to a structural abnormality of the eye [1]. It develops in early childhood and, which is of utmost importance, its timely detection followed by prompt, appropriate treatment can lead to a restoration or enhancement of visual acuity. Recent extensive studies reported a $1.6 \%$ prevalence of amblyopia among preschoolers in the US [2,3]. By and large, due to their extremely busy work schedule, primary care physicians are not consistently conducting pediatric vision screening during routine clinical visits $[4,5]$. In Romania there is currently no established national preschool visual screening system.

The EUSCREEN European project" Implementation of optimized childhood vision and hearing screening programmes in middle-income countries in Europe" brought together distinguished medical centres in Netherlands, United Kingdom, Deutschland, Sweden, Albania and Romania and developed over a 4-year period, between January 2017 and December 2020. The implementation study for visual screening was planned in the Romanian county of Cluj and unfolded over a period of 2 years, between January 2018 and December 2019.

Screening is defined as "a fundamental concept that links clinical practice in individuals, with public health practice in populations" [6]. One of the main concerns of any screening project primarily relate to its cost-efficiency. A recently-published review article on the existing vision screening programmes in 18 countries across 5 continents highlighted "the urgent need for the development of an inexpensive and comprehensive screening tool" [7]. Visual acuity testing in young children performed by eyecare professionals such as ophthalmologists, optometrists revealed very high testability, sensitivity, and specificity (99\%,100\%, and $97 \%$, respectively) [8]. However, the involvement of eyecare professionals in screening is limited due to both a shortage of pediatric ophthalmologists and the limited financial resources of these type of programs. Alternatively, nurses are the healthcare providers generally involved in preventive and health promotion activities. According to a pilot study conducted in Malaysia, involvement of nurses has proved more practical and cost-effective since they are already a part of the health system [9].

Within the EUSCREEN project, visual acuity was measured by medical personnel, either nurses or general practitioners only after completing a mandatory two-day training course organized at the University of Medicine and Pharmacy Cluj-Napoca. Hence, visual screening in the urban areas, namely the city of Cluj-Napoca and the other five municipalities (Turda, Câmpia-Turzii, Huedin, Dej, Gherla) was performed by the nurses in the public kindergartens. In the rural areas, however, visual acuity was tested by the family doctors or their nurses, or, in the final months of the project (and especially in the remote regions of the county) by a travelling nurse employed by the UMF Cluj-Napoca.

This study is a follow-up to our study from 2017 [12] where we evaluated the background knowledge and attitude towards vision screening of the nurses and family doctors potentially enrolled in the project, before the screening activity actually started.

\section{METHOD}

After the ending of the implementation period, the nurses and general practitioners involved in the study were asked to fill in an anonymous 22-questions survey which they received via e-mail or Google Forms. In order to maximize the numbers of replies, the UMF Cluj team had to send the questionnaires twice and even contact the nurses/doctors by phone. 
Still, only 15 out of 55 medical personnel from small municipalities and rural area and 29 doctors and nurses from Cluj-Napoca responded to the survey,. We have briefly analysed the answers in the 44 questionnaires we collected.

\section{RESULTS}

Most of the respondents were nurses; only 1 doctor filled in the questionnaire. Out of the 43 nurses, 24 screened in the city and 11 in one of the small municipalities. The additional 9 nurses performed the examinations in the rural areas, one of them being registered in the project as a travelling nurse.

The analysis of the answers to the first question has revealed the fact that 43 out of the total 44 (97.72\%) medical staff were not enrolled in any other screening programmes on the duration of the EUSCREEN pilot study. Only 1 person $(2.27 \%)$ has taken part in a different screening programme but fails to mention which one.

The second question of the survey was only addressed to the screeners in the rural areas and enquired which would be the best location that would ensure the highest possible screening coverage. Three of the medical personnel opted for the general practitioner's office, while 2 selected the kindergarten as the most appropriate setting for the screening. Moreover, 1 of the respondents did not give any answer. Two others mentioned both the aforementioned possible locations, one of them stating that screening in the kindergarten would enable a higher number of children to be screened, whereas screening in the family doctor's office would guarantee a higher quality of the visual evaluation.

On a 1 to 5 rating scale, with 1 meaning "very insecure" and 5 meaning "very confident", at the end of the 2-year visual screening, the medical staff consider themselves as neutral $(4,54 \%)$, confident $(40,90 \%)$ or very confident $(54,54 \%)$ in their abilities when it comes to the measurement of visual acuity in children.

Regarding the participation rate of children from the locations where they screened, $50 \%$ of the medical staff ( 22 out of 44 ) estimated a screening coverage of $90 \%$ or more; $25 \%$ of the medical staff
(11 out of 44) approximated a ratio between $50 \%$ and $90 \% ; 11.36 \%$ of the medical staff ( 5 out of 44 ) acknowledged a percentage under $50 \%$. An extra $11,36 \%$ (5 out of 44) did not reply to this question and one of the respondents only declared "a high rate", without mentioning the actual percentage.

Among the main reasons why the study did not include a higher number of preschoolers, the participants to the survey stated: lack of parents' consent (8 cases) or lack of time ( 2 cases), interest (3 cases) or confidence in the Euscreen project, some of the parents claiming they would take the children to an ophthalmologist themselves (5 cases); additionally, some of the parents expressed their concern regarding the managing of data confidentiality. Other respondents blamed the poor cooperation with the children because of their small age or claimed that the pre-schoolers in their medical establishment did not belong to the age group eligible for the inclusion in the study (3 cases). Four nurses stressed upon the low attendance rate in kindergartens, one report revealing the fact that rroma ethnic groups usually manifest no interest in their children's education or routine health examinations. One nurse mentioned the children's "fear/anxiety" of being tested. Five respondents declared that all eligible children in their practice were enrolled in the study. Several nurses (12 out of 44) did not reply to this question.

On a scale of 1 to 5 , where 1 means "very reluctant" and 5 means "very open", the participants to the survey consider the parents to be reluctant $(9,09 \%)$, neutral $(6,81 \%)$, open/ enthusiastic $(40,90 \%)$ or even very enthusiastic $(38,63 \%)$ about their children's partaking in a visual acuity screening program.

One of the motivations behind the parents' refusal to give their consent to the participation of their children in the screening was related to their lack of trust in this particular screening program (5 cases). Moreover, some of the parents claimed their pre-schoolers had already been seen by an ophthalmologist (8 responses), whereas others strongly expressed their reluctance towards disclosing personal information, namely the Romanian personal numeric code ( 7 responses). Other reasons mentioned were the 
parents' indolence ( 2 cases), their busy daily schedule (1 case), lack of interest ( 2 responses), lack of financial resources (1 response) or anxiety related to the possible administration of dilating drops (1 response). Additionally, 12 of the respondents argued all the parents consented to the screening; 5 respondents claimed the parents simply did not sign the consent forms and no explanation was provided. 8 nurses did not give any answer to this question.

As to the means that could persuade parents to give their consent for their children's participation in the screening, many respondents $(36,36 \%, 16$ out of 44) consider the key to be a proper information campaign; hence, flyers, audio/video materials could be used for social media marketing; not only nurses and doctors but also teachers could promote the importance of visual screening in an attempt to raise the parents' awareness regarding this issue. Other feasible solutions would be, according to one respondent, the involvement of an ophthalmologist in the visual screening or, according to 2 other respondents, the possibility of the parent to directly observe the visual acuity examination. One nurse suggested complimentary/free pair of glasses in case optical correction is recommended by the ophthalmologist while another advocated for the introduction of a mandatory visual acuity testing prior to the enrolment in kindergarten/ school. Disclosure of personal information should not be required according to one participant in the survey. A significant $25 \%$ (11 out of 44 ) of the respondents stressed upon the fact that the majority if not all the parents agreed to sign the consent and that the current methods employed in the present study are satisfactory and adequate. The question was not answered by 7 nurses.

On a scale of 1 to 5 , with 1 being "very reluctant" and 5 being "very enthusiastic", the respondents considered the children to be neutral $(2,27 \%)$, enthusiastic $(47,72 \%)$ or very enthusiastic $(50 \%)$ about taking part in the project. In this relation, the methods proven to be effective in motivating children to participate in the visual acuity screening were the reward, that is the sticker they received at the end of the examination (24 responses), the friendly presentation of the required task in the form of a challenging game (14 responses). The use of the colourful trial frames (2 responses) as well as viewing of the illustrative animated movie ( 2 responses) prior to the examination also proved helpful. Nonetheless, the fact that in some of the cases the children were already familiar with their examiner ( 2 responses) was invaluable. 1 nurse did not answer this question.

Thirty-seven participants responded to the question directed to the medical personnel in kindergartens. Accordingly, on a scale of 1 to 5, with 1 being "very reluctant" and 5 being "very open", the management of the kindergarten was considered to be open $(21,62 \%$ of them ( 8 out of 37 ) or very open $(78,37 \%, 29$ out of 37) to the involvement in a vision screening programme. When asked the same question with regard to the teachers, the medical staff believed the latter to be neutral $(2,70 \%, 1$ out of 37$)$, open $(24,32 \%, 9$ out of 37$)$ or very open $(72,97 \%, 27$ out of 37$)$ to the children's enrolment in the EUSCREEN project.

The question directed to the nurses in the rural area investigated the most effective methods to attract the target group (children and their legal representatives) from their community in this screening program. According to the 8 nurses who responded, informing the parents on the opportunity of visual screening (which implied no costs for the parents) and explaining the importance of this early detection of ophthalmological problems are essential. One respondent stated that a correct approach would imply seriousness, patience and communication, while another nurse stressed upon the fact that having the teacher's support increased her credibility in front of the parents.

Next, the responders were required to assess the reaction of parents in case of detection of low visual acuity in children. Accordingly, when informed about the necessity of an ophthalmological referral, some parents seemed concerned (8 responses), surprised (1 response) or sceptical (1 response), others appeared appreciative for having been made aware of a visual problem they did not previously suspect (1 response). Many of them (27 responses) showed in favour of an ophthalmological exam that could confirm the 
screening result and hopefully restore visual acuity. However, one of the nurses mentioned a situation in which although the parents took the child to an ophthalmologist, they refused to wear the glasses the child was prescribed.

Among the reasons for declining an ophthalmological exam were the parents' reticence and distrust in the result of the screening ( 2 responses), carelessness, ignorance or lack of involvement in the child's healthcare ( 8 responses), lack of time ( 8 responses), lack of financial resources ( 3 responses). One argued that access to an ophthalmologist was difficult, while other 2 blamed the long waiting list for an ophthalmological appointment. Some parents decided to postpone the examination (1 response), whereas others simply denied the necessity of a visit to the ophthalmologist (3 responses). Five nurses declared they were not given any reasons for the parents' refusal and 3 others stated that all parents who were informed of the test result took their children to the ophthalmologist.

The medical personnel were also asked to rank the priority of the visual acuity screening in the activity of their office on a scale of 1 to 5, with 1 meaning insignificant and 5 meaning very important. Only one nurse $(2,27 \%)$ graded the visual screening as neutral, the majority acknowledging it as either important $(45,45 \%, 20$ out of 44$)$ or very important $(47,72 \%$, 21 out of 44). 2 nurses did not reply to this question. When asked to further discuss their choice, 21 of the respondents emphasised the utmost importance of early detection and appropriate treatment of visual impairments in children. In few cases ( 2 out of 44$)$ the nurses argued that although prevention and detection programs are important, priority should be given to more urgent, acute situations and that screening examinations can be timed.

The question regarding other professionals that should have been included in this screening program only had 32 responders, 21 of whom $(65,62 \%)$ gave a negative and or an uncertain reply (3 responders claiming they do not know the answer to the question). 5 nurses suggested the on site participation of an ophthalmologist could be helpful in the case of parents who refused to attend an ophthalmological exam. Alternatively, two nurses showed in favour of the engagement of a school doctor or general practitioner in the study and one nurse recommended the involvement of the kindergarten teachers who interact with the children on a daily basis and could resort to various educational games in order to persuade children into accepting visual testing more easily.

Nineteen out of the 27 doctors and nurses (70,37\%) that provided an answer to this question consider no other institutions should have been implicated in this screening program. Two nurses suggested the involvement of either the general practitioner's office or of an ophthalmological practice. One respondent suggested that the local town hall could provide the appropriate location for the examination and could help with the dissemination of information to the population (through posters, announcements on the town hall website, advertisements on the town hall's Facebook account and so on). According to one nurse, other options would be the kindergartens or social service agencies as there are certain categories of people that only seldom, if ever, report to the doctor's office.

When asked whether they consider that the involvement of medical staff in such screening programs falls within the duties of the job or if it should always be remunerated in addition to the salary, the answers varied. 15 persons, all working in kindergartens, answered that the visual acuity is usually measured in kindergartens as part of the children's yearly health check-ups and that it is already a part of their job requirements, hence it is already paid for. 19 nurses, from rural areas and from urban kindergartens, consider that working in such screening programmes requires more work, more bureaucracy, therefore an extra payment is needed. 5 persons did not provide an answer to this question, 3 persons abstained. One nurse stated that the answer depends on the nature of the screening, while one rural nurse considers that if the screening takes place in the family doctor's office then it is included in the job requirements, but if the screening would take place in the kindergarten it would require extra working hours and hence extra payment.

We wanted to know what additional measures the EUSCREEN project team should have taken to 
support the screening staff in their activity. 15 nurses considered the Euscreen team organized everything accordingly and did not feel a need for improvement. The other 15 nurses found several aspects they believed required more attention. 7 nurses considered that a higher importance should have been given to the communication with the parents, they thought that more informative materials should have been available and the parents should have been better explained the purpose of the project. 5 nurses demanded there would have been a better collaboration with the ophthalmologists, an easier access for the children to a speciality examination, and one nurse even requested an ophthalmologist trainee to be involved in examining the children in kindergartens when needed. One nurse considered that the screening activity could have been better paid, while another one suggested that the Euscreen team should have assisted and evaluated, randomly, from time to time, the nurse's visual acuity measuring technique. 14 nurses did not provide an answer to this question.

The following question inquired whether the medical staff would continue to measure visual acuity in children even after the completion of the Euscreen programme. 31 respondents are going to continue their screening activity either because it is already part of their job requirements or because they consider that early detection of visual problems in children is crucial. 9 nurses answered that they will not continue the screening activity, invoking reasons such as the fact that they are retired or that they are not working in a kindergarten anymore, or that they found the screening activity arduous due to the reluctance of parents when giving the informed consent; one nurse even added that she would continue only if there was a direct and open collaboration with an ophthalmologist. 4 nurses did not provide an answer to this question.

Next the survey focused on the manner in which the medical staff felt the Euscreen project contributed to their professional development. The majority of the respondents were content to have been a part of this screening project, which they recognized as an enriching experience: they learned many theoretical aspects about vision problems in children, they got to improve their vision acuity measuring technique while also learning to use an eye-chart they never used before (tumbling E's). They noticed they improved their communication skills and their patience, an essential advantage in working with children. A major satisfaction for the nurses was that they were able to detect visual problems at an early age and knowing that their work will have a positive impact on the children's lives. 9 persons did not provide an answer to this question.

The final question of the survey aimed to find the respondents' attitude on the need for a future update regarding the theoretical notions or the practical abilities required for the screening of visual problems. Half of the respondents, 22, feel that such an update is not needed. The other half welcomes the idea of having their notions and skills up to date and 16 of them consider that this should be done once a year $(9$ answers), every two years (6 answers) or every three years (one answer).

\section{DISCUSSION}

In our first study the nurses and the doctors anticipated a fairly large participation rate to the screening, while also foreshadowing potential failures in the implementation process, mainly a poor collaboration and compliance of parents. [12] Two years later, among the main reasons why the study did not include a higher number of preschoolers, the participants to the survey stated: lack of parents' consent or lack of time, interest or confidence in the Euscreen project, some of the parents claiming they would take the children to an ophthalmologist themselves; additionally, some of the parents expressed their concern regarding the managing of data confidentiality. The lack of trust in medical care programmes is cited in literature, mostly because patients do not seem to receive the amount of information they would normally expect about screening [13]. This is because consultations are usually short and information materials cannot be a substitute for good verbal communication [14]. Furthermore, some nurses stressed upon the 
low attendance rate especially in rural kindergartens, where the parents have limited financial and travel means.

Persuading the parents about the importance of the screening is an important topic. The nurses and the doctors felt the supportive materials (flyers, audio video materials) were helpful, and also the teachers' input was of importance in this matter. Still, there were many situations in which the parents did not continue the investigations with an ophthalmological examination, if recommended to, the main reasons being lack of time, lack of interest in the child's healthcare, lack of financial means or too long waiting lists to ophthalmologists. This stresses out the idea that parents should be better informed about the impact amblyopia has on their children's lives.

A small part of the respondents thought that the presence of an ophthalmologist in the kindergarten during the screening days would have erased the parents' doubts and convinced them to allow their children to be examined. Also some of the nurses praised the teachers' role in helping the children not to be scared of the examination.

The topic of reimbursement generated mixed answers. It comes as no surprise that only nurses working in kindergartens feel this type of screening does not need to be extra paid, as visual acuity measurement is included in the children's yearly health-checkups. Still, some kindergarten nurses and the nurses working in family doctor's offices feel that activities performed in a screening programme should be extra paid as they require more work, more time, more bureaucracy. However, literature data shows that paying healthcare providers by the number of persons they pursued to get screened works against the spirit of enabling patients/parents to make an informed choice on whether or not they want them/their children to be screened [16] [17].
Looking back on the two years of screening, half of the respondents considered that everything went according to their expectations, while the other half identified aspects that should have been better, such as the communication with the parents with more informative materials being available, the collaboration with the ophthalmologists and an easier access for the children to a speciality examination. One nurse suggested that the Euscreen team should have assisted and evaluated, randomly, from time to time, the nurses' visual acuity measuring technique.

The medical staff acknowledged that this project contributed to their professional development and feel confident on their new abilities. Only half of the respondents feel that a periodic update on their notions and skills would be needed.

\section{CONCLUSION}

The most important aspect that emerged from studying the questionnaires is that the EUSCREEN project managed to update the theoretical notions and practical abilities of the medical staff, boosted their confidence in the visual acuity examination technique and made them aware of the importance of early detection of vision problems in children, thus the majority of the respondents claiming that they will continue measuring visual acuity in children.

Even if the respondents were not as numerous as for our initial study, their answers provide essential directions that a national screening programme should take into account: a better communication with the parents so that they fully understand the importance of the screening; better access to speciality examinations when needed; better coverage in the rural areas, especially in the remote areas; proper reimbursement for the medical staff. A periodical update of theoretical notions and practical skills would also be welcomed. 


\section{BIBLIOGRAFIE}

1. Braverman Rebecca S. Introduction to amblyopia. Am Acad Ophthalmol 2015 https://www.aao.org/pediatric-center-detail/amblyopia-introduction.

2. USPST Force, Grossman DC, Curry SJ, et al. Vision screening in children aged 6 months to 5 years: US preventive services task force recommendation statement JAMA, 318 (9) (2017), pp. 836-844

3. Shakarchi AF, Collins ME. Referral to community care from school-based eye care programs in the United States. Surv Ophthalmol (2019)

4. Wasserman RC, Croft CA, Brotherton SE. Preschool vision screening in pediatric practice: a study from the Pediatric Research in Office Settings (PROS) Network. Pediatrics. 1992;89(5 Pt 1):834-8

5. Marcinak JF, Yount SC. Evaluation of vision screening practices of Illinois pediatricians. Clin Pediatr. 1995;34:353-7.

6. Speechley M, Kunnilathu A, Aluckal E, Balakrishna MS, Mathew B, George EK.Screening in Public Health and Clinical Care: Similarities and Differences in Definitions, Types, and Aims - A Systematic Review [Internet].2017 March [Cited March1, 2020];11(3):LE01-LE04. Available from:http://www.jcdr.net//back_issues.asp?issn $=0973-709 x \&$ year $=2017 \&$ month $=$ March\&volume $=11 \&$ issue $=3 \&$ page $=$ LE01\&id $=9419$

7. Chen AH, Abu Bakar NF, Arthur P. Comparison of the pediatric vision screening program in 18 countries across five continents. J of Curr Ophthalmology. 2019;31(4); 357-365

8. Bušić $M$, Bjeloš $M$, Petrovečki $M$ et al. Zagreb Amblyopia Preschool Screening Study: near and distance visual acuity testing increase the diagnostic accuracy of screening for amblyopia. Croat Med J. 2016; 57(1): 29-41.

9. Abu Bakar NF, Chen AH, Abdul Rahim MN, Goh PP. Pilot Study: A Review of Personnel Involved in School Vision Screening and the Training Module in Betong, Malaysia. Int Med J Malaysia. 2012;11(2);23-27.

10. Braverman, R. S. (2015). Pediatric vision screening: the Colorado school nurse experience. Journal of American Association for Pediatric Ophthalmology and Strabismus, 19(4), e20.

11. Cockburn J, Redman S, Hill D, Henry E. Public understanding of medical screening. J Med Screen. 1995;2(4):224-7.

12. Vlăduțiu C, Căinap S, Teodosescu $\mathrm{O}$, Ursu RM, Sevan SA, Rajka D, Mara M. Previous expertise and initial attitude towards vision screening in health care professionals enrolled in EUSCREEN project. Journal of School and University Medicine 2020;7(1-2):12-17.

13. Audit Commission. What seems to be the matter: communication between hospitals and patients. London: HMSO; 1993.

14. Coulter A. Evidence based patient information. BMJ. 1998;317:225-226.

15. Entwistle VA, Sheldon TA, Sowden A, Watt IS. Evidence-informed patient choice. Int J Technol Assess Health Care. 1998;14:212-215.

16. Austoker J, McPherson A. Cervical screening. Practical guides for general practice. 2nd ed. Oxford: OUP; 1992. Areas of uncertainty.

17. Austoker J. Gaining informed consent for screening is difficult — but many misconceptions need to be undone. BMJ. 1999 Sep 18; 319(7212): 722-723. 